
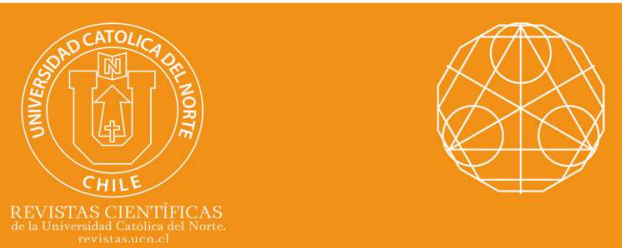

\title{
Trees with vertex-edge roman domination number twice the domination number minus one
}

H. Naresh Kumar ${ }^{1}$ (1) orcid.org/0000-0002-1717-239X

Y. B. Venkatakrishnan ${ }^{2}$ (1) orcid.org/0000-0003-4560-2040

SASTRA University, Dept. of Mathematics, School of Arts, Science and Humanities, Tanjore, TN, India.

1⿴nareshhari1403@gmail.com ; ${ }^{2}$ venkatakrish2@maths.sastra.edu

\section{Abstract:}

$A$ vertex-edge Roman dominating function (or just ve-RDF) of a graph $G=(V, E)$ is a function $f: V(G) \rightarrow\{0,1,2\}$ such that for each edge $e=u v$ either $\max \{f(u), f(v)\} \neq 0$ or there exists a vertex $w$ such that either $w u \in E$ or $w v \in E$ and $f(w)=2$. The weight of a ve-RDF is the sum of its function values over all vertices. The vertex-edge Roman domination number of a graph $G$, denoted by $\gamma_{v e r}(G)$, is the minimum weight of a ve-RDF G. We characterize trees with vertexedge roman domination number equal to twice domination number minus one.

Keywords: Vertex-edge roman dominating set; Dominating set; Trees.

MSC (2020): 05C69.

\section{Cite this article as (IEEE citation style):}

H. Naresh Kumar and Y. B. Venkatakrishnan, "Trees with vertex-edge roman domination number twice the domination number minus one", Proyecciones (Antofagasta, On line), vol. 39, no. 6, pp. 1381-1392, Dec. 2020, doi: 10.22199/issn.07176279-2020-06-0084.

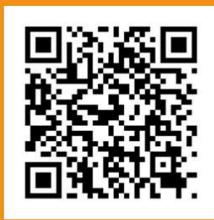

Article copyright: (C) 2020 H. Naresh Kumar and Y. B. Venkatakrishnan. This is an open access article distributed under the terms of the Creative Commons License, which permits unrestricted use and distribution provided the original author and source are credited. 


\section{Introduction}

Let $G=(V, E)$ be a simple graph with order $n=|V|$. For every vertex $v \in V$, the set $\{u \in V \mid u v \in E\}$ denoted by $N(v)$ is the open neighborhood of $v$ and the set $N(v) \cup\{v\}$ is the closed neighborhood of $v$ and is denoted by $N[v]$. The degree of a vertex $v$ is the cardinality of its open neighborhood, denoted $d_{G}(v)=|N(v)|$. A vertex of degree one is called a leaf and its neighbor is called a support vertex. A support vertex is strong (weak, respectively) if it is adjacent to at least two leaves (exactly one leaf, respectively). A star of order $n \geq 2$, denoted by $K_{1, n-1}$, is a tree with at least $n-1$ leaves. A double star is a tree that contains exactly two vertices that are not leaves. A double star with respectively $r$ and $s$ leaves attached to each support vertex is denoted by $D_{r, s}$. The number of edges in a shortest path between vertices $u$ and $v$ is the distance between vertices $u$ and $v$ in a connected graph $G$. The longest distance between any pair of vertices is defined as the diameter of the graph $G$, and is denoted by $\operatorname{diam}(G)$. The edge subdivision for an edge $u v$ is the deletion of $u v$ from $G$ and addition of two edges $u w$ and $w v$ along with the new vertex $w$.

Domination theory is one of the widely studied topic in graph theory. Minimum number of servers in an adhoc network can be modelled as a dominating set of a network graph. A set $S$ of vertices is a dominating set (abbreviated DS) of $G$ if every vertex not in $S$ is adjacent to some vertex in $S$. The minimum cardinality of a dominating set of $G$ is called the domination number, $\gamma(G)$. In domination theory, a set of vertices dominate another set of vertices or a set of edges dominate another set of edges. Peters [7] in 1986 defined a variant of a dominating set in which a set of vertices dominate the edges of a graph $G$. A vertex $v$ ve-dominates every edge incident to a vertex in $N[v]$. A set $S \subseteq V$ is a vertex-edge dominating set (or simply, a ve-dominating set) if for every edge $e \in E$, there exists a vertex $v \in S$ such that $v$ ve-dominates $e$. The minimum cardinality of a $v e$-dominating set of $G$ is called the ve-domination number $\gamma_{v e}(G)$. The concept of vertex-edge domination is studied further in $[2,5,6]$.

A variant of domination, namely Roman dominating function (or just $\mathrm{RDF})$ is a function $f: V(G) \rightarrow\{0,1,2\}$ in which every vertex $u$ for which $f(u)=0$ is adjacent to at least one vertex $v$ for which $f(v)=2$. The weight of an RDF $f$ is $f(V(G))=\sum_{u \in V(G)} f(u)$. The Roman domination number $\gamma_{R}(G)$ is the minimum weight of an $\mathrm{RDF}$ on $G$. For more information on Roman domination and its variants, see $[1,3,4,8,10]$.

In [9], a variation of Roman dominating function and also a variation 
of vertex-edge dominating set, say, vertex-edge Roman dominating function is defined as a function $f: V(G) \rightarrow\{0,1,2\}$ such that each edge $e=v u$ is either incident with a vertex having function value at least one or $u v$ is $v e$-dominated by some vertex $w$ with $f(w)=2$. The vertex-edge Roman domination number $\gamma_{v e R}(G)$ equals the minimum weight of all ve-RDF on $G$.

In this paper we characterize all trees with vertex-edge roman domination number equals twice domination number minus one.

\section{Main results}

We begin the section with an observation.

Observation 1. Let $T^{\prime}$ be a tree with $\gamma_{v e R}\left(T^{\prime}\right)=2 \gamma\left(T^{\prime}\right)-1$. The tree $T$ is obtained from $T^{\prime}$ by attaching a vertex to a support vertex of $T^{\prime}$. Then $\gamma_{v e R}(T)=2 \gamma(T)-1$ if and only if $\gamma_{v e R}\left(T^{\prime}\right)=2 \gamma\left(T^{\prime}\right)-1$.

Proof. Let $x$ be the support vertex in the tree $T^{\prime}$. Let $y$ be the leaf adjacent to $x$. The tree $T$ is obtained from $T^{\prime}$ by adding a vertex $z$ to the vertex $x$. Let $D$ be a $\gamma(T)$-set. To dominate the leaves $y$ and $z$, the vertex $x \in D$. It is easy to see that $D$ is a DS of the tree $T^{\prime}$. Thus $\gamma\left(T^{\prime}\right) \leq \gamma(T)$. Let $D^{\prime}$ be a $\gamma\left(T^{\prime}\right)$-set. If $x \notin D^{\prime}$, then $y \in D^{\prime}$ then obviously $\left(D^{\prime} \backslash\{y\}\right) \cup\{x\}$ is a DS of $T$. Thus $\gamma(T) \leq \gamma\left(T^{\prime}\right)$. We get $\gamma(T)=\gamma\left(T^{\prime}\right)$.

Let $f$ be a $\gamma_{v e R}(T)$-dominating function of the tree $T$. To dominate the edges $x y$ and $x z$, the vertex $x$ is assigned weight one or a vertex in $N(x)$ is assigned weight 2 . If $z$ is assigned weight 2, then the vertex $y$ has weight zero. Replacing the weight of $y$ and $z$, we get $\left.f\right|_{V(T) \backslash\{y\}}$ and $y$ is assigned weight two is a $v e \mathrm{R}$-dominating function of the tree $T^{\prime}$. Thus $\gamma_{v e R}\left(T^{\prime}\right) \leq \gamma_{v e R}(T)$. Let $f^{\prime}$ be a $\gamma_{v e R}\left(T^{\prime}\right)$-dominating function of the tree $T$. To dominate the edge $x y$, the vertex $x$ or $y$ is assigned weight one. If $y$ is assigned weight 1 , then weight of $x$ is zero. Replacing the weight of $x$ and $y$ gives $f$ is a $v e \mathrm{R}$-dominating function of $T$. If weight of $x$ and $y$ are zero, then the vertex which dominates $x y$ dominates $x z$ and in all the cases of $f$ is a $v e \mathrm{R}$-dominating function of $T$. Thus $\gamma_{v e R}(T) \leq \gamma_{v e R}\left(T^{\prime}\right)$. We get $\gamma_{v e R}(T)=\gamma_{v e R}\left(T^{\prime}\right)$. This implies that $\gamma_{v e R}(T)=2 \gamma(T)-1$ if and only if $\gamma_{v e R}\left(T^{\prime}\right)=2 \gamma\left(T^{\prime}\right)-1$.

To prove the required result, we define a family $\mathcal{T}$ containing stars and all trees obtained from a double star by subdividing the edge joining the center twice. We now prove that for every tree of family $\mathcal{T}$, the vertex-edge roman domination number equals the twice domination number minus one. 
Lemma 2. If $T \in \mathcal{T}$, then $\gamma_{v e R}(T)=2 \gamma(T)-1$.

Proof. Let $T \in \mathcal{T}$. If $T$ is $\operatorname{star} K_{1, n}(n \geq 1)$, then $\gamma_{v e R}(T)=1=$ $2(1)-1=2 \gamma(T)-1$. If $T$ is a tree obtained from a double star by subdividing the edge joining the center twice, then the support vertices is a minimum dominating set. Thus $\gamma(T)=2$. If two support vertices are assigned weight one and two respectively, then we have $\gamma_{v e R}(T)=3$. We get $\gamma_{v e R}(T)=3=2(2)-1=2 \gamma(T)-1$.

We now prove that if vertex-edge roman domination number of a tree is equal to twice domination number minus one, then the tree belongs to the family $\mathcal{T}$.

Lemma 3. For every tree $T, \gamma_{v e R}(T)=2 \gamma(T)-1$, then $T \in \mathcal{T}$

Proof. First assume that $\operatorname{diam}(T)=1$. The tree $T=K_{2}$. We have $\gamma_{v e R}(T)=1=2 \gamma(T)-1$. Thus $T \in \mathcal{T}$. Now assume that $\operatorname{diam}(T)=2$. The tree $T$ is a star. We have $\gamma_{v e R}(T)=2 \gamma(T)-1$. Thus $T \in \mathcal{T}$. Now assume that $\operatorname{diam}(T) \geq 3$. Thus the order $n$ of the tree $T$ is at least four. The result we obtain by the induction on the number $n$. Assume that the theorem is true for every tree $T^{\prime}$ of order $n^{\prime}<n$.

We now root $T$ at a vertex $r$ of maximum eccentricity $\operatorname{diam}(T)$. Let $t$ be a leaf at maximum distance from $r, v$ be the parent of $t, u$ be the parent of $v, w$ be the parent of $u, d$ be the parent of $w$ and $e$ be the parent of $d$ in the rooted tree. By $T_{x}$ we denote the subtree induced by a vertex $x$ and its descendants in the rooted tree $T$.

By Observation 1, we can assume that $d_{T}(v)=2$. Assume that among the children of $u$ there is a support vertex, say $x$, other than $v$. The leaf adjacent to $x$ we denote by $y$. Let $T^{\prime}=T-T_{v}$. Let $f_{1}$ be a $\gamma_{v e R}\left(T^{\prime}\right)$ dominating function of tree $T^{\prime}$. The function $f$ defined on $V(T)$ as

$$
f(a)=\left\{\begin{array}{cll}
f_{1}(a) & \text { if } & a \in V\left(T^{\prime}\right) \\
1 & \text { if } & a=v \\
0 & \text { if } & \text { otherwise }
\end{array}\right.
$$

is a $v e R$-dominating function of $T$. Thus $\gamma_{v e R}(T) \leq \gamma_{v e R}\left(T^{\prime}\right)+1$. Let $D$ be a $\gamma(T)$-set. To dominate the vertices $t$ and $y$, the vertices $v, x \in D$. It is easy to observe that $D \backslash\{v\}$ is a DS of $T^{\prime}$. Thus $\gamma\left(T^{\prime}\right) \leq \gamma(T)-1$. We have $\gamma_{v e R}(T) \leq \gamma_{v e R}\left(T^{\prime}\right)+1 \leq 2 \gamma\left(T^{\prime}\right)-1+1 \leq 2(\gamma(T)-1)<2 \gamma(T)-1$.

Now assume that among the children of $u$, say $x$, is a leaf. Let $T^{\prime}=$ $T-T_{v}$. Let $f_{1}$ be a $\gamma_{v e R}\left(T^{\prime}\right)$-dominating function of the tree $T^{\prime}$. The function $f$ defined on $V(T)$ as 


$$
f(a)=\left\{\begin{array}{cll}
f_{1}(a) & \text { if } & a \in V\left(T^{\prime}\right) \\
1 & \text { if } & a=v \\
0 & \text { if } & \text { otherwise }
\end{array}\right.
$$

is a $v e R$-dominating function of $T$. Thus $\gamma_{v e R}(T) \leq \gamma_{v e R}\left(T^{\prime}\right)+1$. Let $D$ be a $\gamma(T)$-set. To dominate the vertices $t$ and $x$, the vertices $v, u \in D$. It is easy to observe that $D \backslash\{v\}$ is a DS of $T^{\prime}$. Thus $\gamma\left(T^{\prime}\right) \leq \gamma(T)-1$. We have $\gamma_{v e R}(T) \leq \gamma_{v e R}\left(T^{\prime}\right)+1 \leq 2 \gamma\left(T^{\prime}\right)-1+1 \leq 2(\gamma(T)-1)<2 \gamma(T)-1$.

Assume that $d_{T}(u)=2$. Now assume that among the children of $w$, other than $u$, say $x$, such that the distance of $w$ to the most distant vertex of $T_{x}$ is three. It suffices to consider the case $T_{x}=P_{3}=x y z$. Let $T^{\prime}=T-T_{u}$. Let $f_{1}$ be a $\gamma_{v e R}\left(T^{\prime}\right)$-dominating function of the tree $T^{\prime}$. To dominate the edges $z y$ and $x y$, the vertex $x$ is assigned weight two. The function $f$ defined on $V(T)$ as

$$
f(a)=\left\{\begin{array}{cll}
f_{1}(a) & \text { if } & a \in V\left(T^{\prime}\right) \\
1 & \text { if } & a=v \\
0 & \text { if } & \text { otherwise }
\end{array}\right.
$$

is a $v e R$-dominating function of $T$. Thus $\gamma_{v e R}(T) \leq \gamma_{v e R}\left(T^{\prime}\right)+1$. Let $D$ be a $\gamma(T)$-set. To dominate the vertices $t$ and $z$, the vertices $v, y \in D$. It is easy to observe that $D \backslash\{v\}$ is a DS of $T^{\prime}$. Thus $\gamma\left(T^{\prime}\right) \leq \gamma(T)-1$. We have $\gamma_{v e R}(T) \leq \gamma_{v e R}\left(T^{\prime}\right)+1 \leq 2 \gamma\left(T^{\prime}\right)-1+1 \leq 2(\gamma(T)-1)<2 \gamma(T)-1$.

Assume that among the children of $w$, other than $u$, say $x$, such that the distance of $w$ to the most distant vertex of $T_{x}$ is two. It suffices to consider the case $T_{x}=P_{2}=x y$. Let $T^{\prime}=T-T_{u}$. Let $f_{1}$ be a $\gamma_{v e R}\left(T^{\prime}\right)$-dominating function of the tree $T^{\prime}$. To dominate the edges $x y$ and $w x$, the vertex $w$ is assigned weight two. The function $f$ defined on $V(T)$ as

$$
f(a)=\left\{\begin{array}{cll}
f_{1}(a) & \text { if } & a \in V\left(T^{\prime}\right) \\
1 & \text { if } & a=v \\
0 & \text { if } & \text { otherwise }
\end{array}\right.
$$

is a $v e R$-dominating function of $T$. Thus $\gamma_{v e R}(T) \leq \gamma_{v e R}\left(T^{\prime}\right)+1$. Let $D$ be a $\gamma(T)$-set. To dominate the vertices $t$ and $y$, the vertices $v, x \in D$. It is easy to observe that $D \backslash\{v\}$ is a DS of $T^{\prime}$. Thus $\gamma\left(T^{\prime}\right) \leq \gamma(T)-1$. We have $\gamma_{v e R}(T) \leq \gamma_{v e R}\left(T^{\prime}\right)+1 \leq 2 \gamma\left(T^{\prime}\right)-1+1 \leq 2(\gamma(T)-1)<2 \gamma(T)-1$.

Assume that among the children of $w$, say $x$ is a leaf. Let $T^{\prime}=T-T_{u}$. Let $f_{1}$ be a $\gamma_{v e R}\left(T^{\prime}\right)$-dominating function of the tree $T^{\prime}$. The vertex which dominates the edge $w x$ in tree $T^{\prime}$ dominates the edge $w u$ in the tree $T$. The function $f$ defined on $V(T)$ as 


$$
f(a)=\left\{\begin{array}{cll}
f_{1}(a) & \text { if } & a \in V\left(T^{\prime}\right) \\
1 & \text { if } & a=v \\
0 & \text { if } & \text { otherwise }
\end{array}\right.
$$

is a $v e R$-dominating function of $T$. Thus $\gamma_{v e R}(T) \leq \gamma_{v e R}\left(T^{\prime}\right)+1$. Let $D$ be a $\gamma(T)$-set. To dominate the vertices $t$ and $x$, the vertices $v, w \in D$. It is easy to observe that $D \backslash\{v\}$ is a DS of $T^{\prime}$. Thus $\gamma\left(T^{\prime}\right) \leq \gamma(T)-1$. We have $\gamma_{v e R}(T) \leq \gamma_{v e R}\left(T^{\prime}\right)+1 \leq 2 \gamma\left(T^{\prime}\right)-1+1 \leq 2(\gamma(T)-1)<2 \gamma(T)-1$.

Assume that among the children of $d$, other than $w$, say $x$, such that the distance of $d$ to the most distant vertex of $T_{x}$ is three. It suffices to consider the case $T_{x}=P_{3}=x y z$. Let $T^{\prime}=T-T_{w}-T_{y}$. Let $f_{1}$ be a $\gamma_{v e R}\left(T^{\prime}\right)$-dominating function of the tree $T^{\prime}$. The function $f$ defined on $V(T)$ as

$$
f(a)=\left\{\begin{array}{cll}
f_{1}(a) & \text { if } & a \in V\left(T^{\prime}\right) \\
1 & \text { if } & a=y \\
2 & \text { if } & a=u \\
0 & \text { if } & \text { otherwise }
\end{array}\right.
$$

is a $v e R$-dominating function of $T$. Thus $\gamma_{v e R}(T) \leq \gamma_{v e R}\left(T^{\prime}\right)+3$. Let $D$ be a $\gamma(T)$-set. To dominate the vertices $w, u, t, x$ and $z$, the vertices $d, v, y \in D$. It is easy to observe that $D \backslash\{v, y\}$ is a DS of $T^{\prime}$. Thus $\gamma\left(T^{\prime}\right) \leq \gamma(T)-2$. We have $\gamma_{v e R}(T) \leq \gamma_{v e R}\left(T^{\prime}\right)+3 \leq 2 \gamma\left(T^{\prime}\right)-1+3 \leq 2(\gamma(T)-1)<2 \gamma(T)-1$.

Assume that among the children of $d$, other than $w$, say $x$, such that the distance of $d$ to the most distant vertex of $T_{x}$ is two. It suffices to consider the case $T_{x}=P_{2}=x y$. Let $T^{\prime}=T-T_{w}-y$. Let $f_{1}$ be a $\gamma_{v e R}\left(T^{\prime}\right)$-dominating function of the tree $T^{\prime}$. The function $f$ defined on $V(T)$ as

$$
f(a)=\left\{\begin{array}{cll}
f_{1}(a) & \text { if } & a \in V\left(T^{\prime}\right) \\
1 & \text { if } & a=x \\
2 & \text { if } & a=u \\
0 & \text { if } & \text { otherwise }
\end{array}\right.
$$

is a $v e R$-dominating function of $T$. Thus $\gamma_{v e R}(T) \leq \gamma_{v e R}\left(T^{\prime}\right)+3$. Let $D$ be a $\gamma(T)$-set. To dominate the vertices $w, u, t$ and $y$, the vertices $d, v, x \in D$. It is easy to observe that $D \backslash\{v, x\}$ is a DS of $T^{\prime}$. Thus $\gamma\left(T^{\prime}\right) \leq \gamma(T)-2$. We have $\gamma_{v e R}(T) \leq \gamma_{v e R}\left(T^{\prime}\right)+3 \leq 2 \gamma\left(T^{\prime}\right)-1+3 \leq 2(\gamma(T)-1)<2 \gamma(T)-1$.

Assume that among the children of $d$, other than $w$, say $x$, such that the distance of $w$ to the most distant vertex of $T_{x}$ is four. It suffices to consider the tree $T_{x}=P_{4}=x y z a$. We fix $d_{T}(d)=3$. Assume that $e$ is adjacent to 
$T_{k}$, where $T_{k}$ is isomorphic to $T_{d}$ or $T_{k}$ is $P_{5}$ or $P_{2}$. Let $T^{\prime}=T-T_{d}$. Let $f_{1}$ be a $\gamma_{v e R}\left(T^{\prime}\right)$-dominating function of the tree $T^{\prime}$. The function $f$ defined on $V(T)$ as

$$
f(c)=\left\{\begin{array}{cll}
f_{1}(c) & \text { if } & c \in V\left(T^{\prime}\right) \\
2 & \text { if } & c=d \\
1 & \text { if } & c=v, z \\
0 & \text { if } & \text { otherwise }
\end{array}\right.
$$

is a $v e R$-dominating function of $T$. Thus $\gamma_{v e R}(T) \leq \gamma_{v e R}\left(T^{\prime}\right)+4$. Let $D$ be a $\gamma(T)$-set. To dominate the vertices $e, w, x, u, t, y$ and $a$, the vertices $d, v, z \in D$. Since $k \in D, D \backslash\{d, v, z\}$ is a DS of $T^{\prime}$. Thus $\gamma\left(T^{\prime}\right) \leq \gamma(T)-3$. We have $\gamma_{v e R}(T) \leq \gamma_{v e R}\left(T^{\prime}\right)+4 \leq 2 \gamma\left(T^{\prime}\right)-1+4 \leq 2(\gamma(T)-3)+3<$ $2 \gamma(T)-1$.

Now assume that $T_{k}$ is $P_{4}$ or $P_{1}$. Let $T^{\prime}=T-T_{d}$. Let $f_{1}$ be a $\gamma_{v e R}\left(T^{\prime}\right)$ dominating function of the tree $T^{\prime}$. The function $f$ defined on $V(T)$ as

$$
f(c)=\left\{\begin{array}{cll}
f_{1}(c) & \text { if } & c \in V\left(T^{\prime}\right) \\
2 & \text { if } & c=d \\
1 & \text { if } & c=v, z \\
0 & \text { if } & \text { otherwise }
\end{array}\right.
$$

is a $v e R$-dominating function of $T$. Thus $\gamma_{v e R}(T) \leq \gamma_{v e R}\left(T^{\prime}\right)+4$. Let $D$ be a $\gamma(T)$-set. To dominate the vertices $w, x, u, y, t$ and $a$, the vertices $d, v, y \in$ $D$. Since $e \in D, D \backslash\{d, v, z\}$ is a DS of $T^{\prime}$. Thus $\gamma\left(T^{\prime}\right) \leq \gamma(T)-3$. We have $\gamma_{v e R}(T) \leq \gamma_{v e R}\left(T^{\prime}\right)+4 \leq 2 \gamma\left(T^{\prime}\right)-1+4 \leq 2(\gamma(T)-3)+3<2 \gamma(T)-1$.

Suppose $e$ is adjacent to two $P_{3}$, say $k l m$ and $k_{1} l_{1} m_{1}$. Let $T^{\prime}=T-T_{k}$. It is easy to check that $\gamma_{v e R}(T)<2 \gamma(T)-1$. Now assume that $e$ is adjacent to exactly one $P_{3}=k l m$. Let $T^{\prime}=T-T_{e}$. Let $f_{1}$ be a $\gamma_{v e R}\left(T^{\prime}\right)$-dominating function of the tree $T^{\prime}$. The function $f$ defined on $V(T)$ as

$$
f(c)=\left\{\begin{array}{cll}
f_{1}(c) & \text { if } & c \in V\left(T^{\prime}\right) \\
2 & \text { if } & c=d \\
1 & \text { if } & c=v, z, l \\
0 & \text { if } & \text { otherwise }
\end{array}\right.
$$

is a $v e R$-dominating function of $T$. Thus $\gamma_{v e R}(T) \leq \gamma_{v e R}\left(T^{\prime}\right)+5$. Let $D$ be a $\gamma(T)$-set. To dominate the vertices $e, w, x, u, y, t, a, k$ and $m$, the vertices $d, v, z, l \in D$. It is easy to observe that $D \backslash\{d, v, z, l\}$ is a DS of $T^{\prime}$. Thus $\gamma\left(T^{\prime}\right) \leq \gamma(T)-4$. We have $\gamma_{v e R}(T) \leq \gamma_{v e R}\left(T^{\prime}\right)+5 \leq 2 \gamma\left(T^{\prime}\right)-1+5 \leq$ $2(\gamma(T)-4)+4<2 \gamma(T)-1$. 
Now assume that $d_{T}(e)=2$. Let $T^{\prime}=T-T_{e}$. Let $f_{1}$ be a $\gamma_{v e R}\left(T^{\prime}\right)$ dominating function of the tree $T^{\prime}$. The function $f$ defined on $V(T)$ as

$$
f(c)=\left\{\begin{array}{cll}
f_{1}(c) & \text { if } & c \in V\left(T^{\prime}\right) \\
2 & \text { if } & c=d \\
1 & \text { if } & c=v, z \\
0 & \text { if } & \text { otherwise }
\end{array}\right.
$$

is a $v e R$-dominating function of $T$. Thus $\gamma_{v e R}(T) \leq \gamma_{v e R}\left(T^{\prime}\right)+4$. Let $D$ be a $\gamma(T)$-set. To dominate the vertices $e, w, x, u, y, t$ and $a$, the vertices $d, v, z \in D$. It is easy to observe that $D \backslash\{d, v, z\}$ is a DS of $T^{\prime}$. Thus $\gamma\left(T^{\prime}\right) \leq \gamma(T)-3$. We have $\gamma_{v e R}(T) \leq \gamma_{v e R}\left(T^{\prime}\right)+4 \leq 2 \gamma\left(T^{\prime}\right)-1+4 \leq$ $2(\gamma(T)-3)+3<2 \gamma(T)-1$.

Assume among the children of $d$, say $h$, is a leaf. We have $d_{T}(d)=3$. Assume $e$ is adjacent to $e$ say $T_{k}$, where $T_{k}$ is isomorphic to $T_{d}$ or $T_{k}$ is $P_{5}$ or $P_{2}$. Let $T^{\prime}=T-T_{d}$. Let $f_{1}$ be a $\gamma_{v e R}\left(T^{\prime}\right)$-dominating function of the tree $T^{\prime}$. The function $f$ defined on $V(T)$ as

$$
f(a)=\left\{\begin{array}{cll}
f_{1}(a) & \text { if } & a \in V\left(T^{\prime}\right) \\
2 & \text { if } & a=u \\
1 & \text { if } & a=d \\
0 & \text { if } & \text { otherwise }
\end{array}\right.
$$

is a $v e R$-dominating function of $T$. Thus $\gamma_{v e R}(T) \leq \gamma_{v e R}\left(T^{\prime}\right)+3$. Let $D$ be a $\gamma(T)$-set. To dominate the vertices $h, w, u, t$ and $e$, the vertices $v, d \in D$. Since $k \in D, D \backslash\{d, v\}$ is a DS of $T^{\prime}$. Thus $\gamma\left(T^{\prime}\right) \leq \gamma(T)-2$. We have $\gamma_{v e R}(T) \leq \gamma_{v e R}\left(T^{\prime}\right)+3 \leq 2 \gamma\left(T^{\prime}\right)-1+3 \leq 2(\gamma(T)-1)<2 \gamma(T)-1$.

Now assume that $T_{k}$ is $P_{4}$ or $P_{1}$. Let $T^{\prime}=T-T_{d}$. Let $f_{1}$ be a $\gamma_{v e R}\left(T^{\prime}\right)$ dominating function of the tree $T^{\prime}$. The function $f$ defined on $V(T)$ as

$$
f(a)=\left\{\begin{array}{cll}
f_{1}(a) & \text { if } & a \in V\left(T^{\prime}\right) \\
2 & \text { if } & a=u \\
1 & \text { if } & a=d \\
0 & \text { if } & \text { otherwise }
\end{array}\right.
$$

is a $v e R$-dominating function of $T$. Thus $\gamma_{v e R}(T) \leq \gamma_{v e R}\left(T^{\prime}\right)+3$. Let $D$ be a $\gamma(T)$-set. To dominate the vertices $h, w, u$ and $t$, the vertices $v, d \in D$. Since $e \in D, D \backslash\{d, v\}$ is a DS of $T^{\prime}$. Thus $\gamma\left(T^{\prime}\right) \leq \gamma(T)-2$. We have $\gamma_{v e R}(T) \leq \gamma_{v e R}\left(T^{\prime}\right)+3 \leq 2 \gamma\left(T^{\prime}\right)-1+3 \leq 2(\gamma(T)-1)<2 \gamma(T)-1$.

Now assume that $T_{k}$ is $P_{3}=x y z$. Let $T^{\prime}=T-T_{x}$. Let $f_{1}$ be a $\gamma_{v e R}\left(T^{\prime}\right)$ dominating function of the tree $T^{\prime}$. To dominate the edges $d w, w v, v u, u t$ 
the vertex $v$ is assigned a weight 2 and to dominate the edges $h d$, de vertex $e$ is assigned weight 2 . The function $f$ defined on $V(T)$ as

$$
f(a)=\left\{\begin{array}{cll}
f_{1}(a) & \text { if } & a \in V\left(T^{\prime}\right) \\
2 & \text { if } & a=y \\
0 & \text { if } & \text { otherwise }
\end{array}\right.
$$

is a $v e R$-dominating function of $T$. Thus $\gamma_{v e R}(T) \leq \gamma_{v e R}\left(T^{\prime}\right)+1$. Let $D$ be a $\gamma(T)$-set. To dominate the vertices $x$ and $z$, the vertex $y \in D$. It is easy to observe that $D \backslash\{y\}$ is a DS of $T^{\prime}$. Thus $\gamma\left(T^{\prime}\right) \leq \gamma(T)-1$. We have $\gamma_{v e R}(T) \leq \gamma_{v e R}\left(T^{\prime}\right)+1 \leq 2 \gamma\left(T^{\prime}\right)-1+1 \leq 2(\gamma(T)-1)<2 \gamma(T)-1$.

Now assume that $d_{T}(e)=2$. Let Let $T^{\prime}=T-T_{e}$. Let $f_{1}$ be a $\gamma_{v e R}\left(T^{\prime}\right)$ dominating function of the tree $T^{\prime}$. The function $f$ defined on $V(T)$ as

$$
f(a)=\left\{\begin{array}{cll}
f_{1}(a) & \text { if } & a \in V\left(T^{\prime}\right) \\
2 & \text { if } & a=d \\
1 & \text { if } & a=v \\
0 & \text { if } & \text { otherwise }
\end{array}\right.
$$

is a $v e R$-dominating function of $T$. Thus $\gamma_{v e R}(T) \leq \gamma_{v e R}\left(T^{\prime}\right)+3$. Let $D$ be a $\gamma(T)$-set. To dominate the vertices $e, h, w, u, v$ and $t$, the vertices $v, d \in D$. It is easy to observe that $D \backslash\{d, v\}$ is a DS of $T^{\prime}$. Thus $\gamma\left(T^{\prime}\right) \leq \gamma(T)-2$. We have $\gamma_{v e R}(T) \leq \gamma_{v e R}\left(T^{\prime}\right)+3 \leq 2 \gamma\left(T^{\prime}\right)-1+3 \leq 2(\gamma(T)-1)<2 \gamma(T)-1$.

Now assume $d_{T}(d)=2$. Assume that some child of $e$, say $x$, other than $d$ such that the distance of $e$ to the most distant vertex of $T_{x}$ is five or two. It suffices to consider the case $T_{x}=P_{5}=x y z a b$ or $T_{x}=P_{2}=x y$. Let $T^{\prime}=T-T_{d}$. Let $f_{1}$ be a $\gamma_{v e R}\left(T^{\prime}\right)$-dominating function of the tree $T^{\prime}$. The function $f$ defined on $V(T)$ as

$$
f(a)=\left\{\begin{array}{cll}
f_{1}(a) & \text { if } & a \in V\left(T^{\prime}\right) \\
2 & \text { if } & a=u \\
1 & \text { if } & a=d \\
0 & \text { if } & \text { otherwise }
\end{array}\right.
$$

is a $v e R$-dominating function of $T$. Thus $\gamma_{v e R}(T) \leq \gamma_{v e R}\left(T^{\prime}\right)+3$. Let $D$ be a $\gamma(T)$-set. To dominate the vertices $w, u$ and $t$, the vertices $d, v \in D$. The vertex $e$ is adjacent to $P_{5}$ or $P_{2}$, the child of $e$ other than $d$ is in $D$. It is easy to observe that $D \backslash\{v, d\}$ is a DS of $T^{\prime}$. Thus $\gamma\left(T^{\prime}\right) \leq \gamma(T)-2$. We have $\gamma_{v e R}(T) \leq \gamma_{v e R}\left(T^{\prime}\right)+3 \leq 2 \gamma\left(T^{\prime}\right)-1+3 \leq 2(\gamma(T)-1)<2 \gamma(T)-1$.

Assume that some child of $e$, say $x$, other than $d$ such that the distance of $e$ to the most distant vertex of $T_{x}$ is four or one. It suffices to consider the case $T_{x}=P_{4}=x y z a$ or $T_{x}=P_{1}=x$. Let $T^{\prime}=T-T_{w}$. Let $f_{1}$ be 
a $\gamma_{v e R}\left(T^{\prime}\right)$-dominating function of the tree $T^{\prime}$. The function $f$ defined on $V(T)$ as

$$
f(a)=\left\{\begin{array}{cll}
f_{1}(a) & \text { if } & a \in V\left(T^{\prime}\right) \\
2 & \text { if } & a=u \\
0 & \text { if } & \text { otherwise }
\end{array}\right.
$$

is a $v e R$-dominating function of $T$. Thus $\gamma_{v e R}(T) \leq \gamma_{v e R}\left(T^{\prime}\right)+2$. Let $D$ be a $\gamma(T)$-set. To dominate the vertices $w, u$ and $t$, the vertices $d, v \in D$. The vertex $e$ is adjacent to $P_{4}$ or $P_{1}$. To dominate the children of $e$ other than $d$, the vertex $e$ is in $D$. It is easy to observe that $D \backslash\{v, d\}$ is a DS of $T^{\prime}$. Thus $\gamma\left(T^{\prime}\right) \leq \gamma(T)-2$. We have $\gamma_{v e R}(T) \leq \gamma_{v e R}\left(T^{\prime}\right)+2 \leq 2 \gamma\left(T^{\prime}\right)-1+2<$ $2 \gamma(T)-1$.

Assume that some child of $e$, say $x$, other than $d$ such that the distance of $e$ to the most distant vertex of $T_{x}$ is three. It suffices to consider the case $T_{x}=P_{3}=x y z$. Let $T^{\prime}=T-T_{x}$. Let $f_{1}$ be a $\gamma_{v e R}\left(T^{\prime}\right)$-dominating function of the tree $T^{\prime}$. To dominate the edges $v t, u v$ the vertex $u$ is assigned weight two. To dominate the edge $e d$, we assign a weight one to the vertex $e$ or two to the vertex $f$. This vertex dominates the edge $e x$ in the tree $T$. The function $f$ defined on $V(T)$ as

$$
f(a)=\left\{\begin{array}{cll}
f_{1}(a) & \text { if } & a \in V\left(T^{\prime}\right) \\
1 & \text { if } & a=y \\
0 & \text { if } & \text { otherwise }
\end{array}\right.
$$

is a $v e R$-dominating function of $T$. Thus $\gamma_{v e R}(T) \leq \gamma_{v e R}\left(T^{\prime}\right)+1$. Let $D$ be a $\gamma(T)$-set. To dominate the vertex $z$, the vertex $y \in D$. It is clear that $D \backslash\{y\}$ is a DS of $T^{\prime}$. Thus $\gamma\left(T^{\prime}\right) \leq \gamma(T)-1$. We have $\gamma_{v e R}(T) \leq$ $\gamma_{v e R}\left(T^{\prime}\right)+1 \leq 2 \gamma\left(T^{\prime}\right)<2 \gamma(T)-1$.

Assume that $d_{T}(e)=1$. We have $T=P_{6}$. Then $\gamma_{v e R}\left(P_{6}\right)=3=$ $2(2)-1=2 \gamma\left(P_{6}\right)-1, T \in \mathcal{T}$. Now assume that $d_{T}(e)=2$. Let $T^{\prime}=T-T_{e}$. Let $f_{1}$ be a $\gamma_{v e R}\left(T^{\prime}\right)$-dominating function of the tree $T^{\prime}$. To dominate the edges $v t, u v$ the vertex $u$ is assigned weight two. To dominate the edge $e d$, we assign a weight one to the vertex $e$. The function $f$ defined on $V(T)$ as

$$
f(a)=\left\{\begin{array}{cll}
f_{1}(a) & \text { if } & a \in V\left(T^{\prime}\right) \\
1 & \text { if } & a=e \\
2 & \text { if } & a=u \\
0 & \text { if } & \text { otherwise }
\end{array}\right.
$$

is a $v e R$-dominating function of $T$. Thus $\gamma_{v e R}(T) \leq \gamma_{v e R}\left(T^{\prime}\right)+3$. Let $D$ be a $\gamma(T)$-set. To dominate the vertices $t$ and $w$, the vertices $v, d \in D$. It 
is obvious that $D \backslash\{v, d\}$ is a DS of $T^{\prime}$. Thus $\gamma\left(T^{\prime}\right) \leq \gamma(T)-2$. We have $\gamma_{v e R}(T) \leq \gamma_{v e R}\left(T^{\prime}\right)+3 \leq 2 \gamma\left(T^{\prime}\right)-1+3 \leq 2 \gamma\left(T^{\prime}\right)+2<2 \gamma(T)-1$.

As a immediate consequence of Lemmas 2 and 3, we have the following characterization of trees with vertex-edge roman domination number equal to twice domination number minus one.

Theorem 4. Let $T$ be a tree. Then $\gamma_{v e R}(T)=2 \gamma(T)-1$ if and only if $T \in \mathcal{T}$.

\section{Acknowledgement}

The second author thanks DST SERB(MATRICS)- grant number MTR/ 2018/000234 for the support.

\section{References}

[1] H. A. Ahangar, J. Amjadi, M. Chellali, S. Nazari-Moghaddam, and S. M. Heikholeslami, "Trees with double roman domination number twice the domination number plus two", Iranian journal of science and technology, transactions A: Science volume, vol. 43, pp. 1081-1088, Jun. 2019, doi: 10.1007/ s40995-018-0535-7

[2] R. Boutrig, M. Chellali, T. W. Haynes, and S. T. Hedetniemi, "Vertex-edge domination in graphs", Aequationes mathematicae, vol. 90, no. 2, pp. 355-366, May 2015, doi: 10.1007/ s00010-015-0354-2

[3] M. Chellali and N. J. Rad, "Trees with unique Roman dominating functions of minimum weight", Discrete mathematics, algorithms and applications, vol. 06, no. 03, Art ID 1450038, Jun. 2014., doi: 10.1142/ S1793830914500384

[4] E. J. Cockayne, P. A. Dreyer, S. M. Hedetniemi, and S. T. Hedetniemi, "Roman domination in graphs", Discrete mathematics, vol. 278, no. 1-3, pp. 11-22, Mar. 2004, doi: 10.1016/j.disc.2003.06.004

[5] B. Krishnakumari, Y. B. Venkatakrishnan, and M. Krzywkowski, "Bounds on the vertex-edge domination number of a tree", Comptes rendus mathematique, vol. 352, no. 5, pp. 363-366, May 2014, doi: 10.1016/j.crma.2014.03.017

[6] J. R. Lewis, S. T. Hedetniemi, T. W. Haynes, and G. H. Fricke, "Vertexedge domination", Utilitas mathematica, vol. 81, pp. 193-213, 2010. 
[7] J. W. Peters, "Theoretical and algorithmic results on domination and connectivity", Ph. D. Thesis, Clemson University, 1986. [On line]. Available: https:/ / bit.ly/ 34lu9SS

[8] P. R. L. Pushpam and S. Padmapriea, "Global Roman domination in graphs", Discrete applied mathematics, vol. 200, pp. 176-185, Feb. 2016, doi: 10.1016/j.dam.2015.07.014

[9] E. N. Satheesh, "Some variations of domination and applications", Ph. D. Thesis, Mahatma Gandhi University, 2014. [On line]. Available: https:/ / bit.ly/ 2HvoErI

[10] X. Zhang, Z. Li, H. Jiang, and Z. Shao, “Double Roman domination in trees", Information processing letters, vol. 134, pp. 31-34, Jun. 2018, doi: $10.1016 /$ j.ipl.2018.01.004 